\title{
PODCASTING EN LA EDUCACIÓN SUPERIOR. UNA APROXIMACIÓN AL ÁMBITO HISPANOAMERICANO
}

\section{PODCASTING IN HIGHER EDUCATION. AN APPROACH TO SPANISH AMERICA SITUATION}

\section{AUTORA}

Ma Teresa Piñeiro Otero.

Universidad de Vigo.

m.terepo@ hotmail.com

\section{RESUMEN}

La incorporación de las TICs al ámbito de la educación ha supuesto la flexibilización de las instituciones educativas, al tiempo que ha favorecido el desarrollo del proceso de enseñanza-aprendizaje.

Entre las herramientas de la web 2.0 que ofrecen mayor potencial en el contexto educativo resultan de particular interés los podcast. Unos archivos sonoros sindicados que, a pesar de su reciente aparición, han vivido una importante expansión en el ámbito de las estrategias didácticas.

El objeto del presente trabajo ha sido el de realizar una aproximación al fenómeno de los podcast en el contexto de la educación superior, con especial atención en lo que respecta a las primeras iniciativas que -sobre este ámbitose han desarrollado en Hispanoamérica. 
TIC - Podcast - Educación superior

\section{ABSTRACT}

ICT incorporation to education field has become to the educational institutions more flexible and it has favoured education-learning process development.

Among most potential web 2.0 tools, in an educational context, podcast is particularly interesting. Some audible files that, in spite of its recent apparition, have lived an important expansion in didactic strategies field.

Present work aims to demonstrate the phenomenon approximation in higher education context, with special attention to this kind of initiatives in Spanish America context.

\section{KEY WORDS}

ICT - Podcasting - Higher education

\section{ÍNDICE}

1. Introducción

2. Aproximación al concepto de podcast

3. Posibilidades para la educación

4. Experiencias pioneras de podcasting en la educación superior

5. Principales iniciativas en el ámbito hispanoamericano

6. Los podcast en la educación. Algunas conclusiones

7. Bibliografía

8. Webgrafía

\section{Introducción}


En 2005 el New Oxford American Diccionary seleccionó el término "podcasting" como palabra del año. Esta declaración, apenas un año después de la creación del primer podcast, constituye el mejor ejemplo para ilustrar la rápida eclosión de este fenómeno en la vida cotidiana de miles de personas, así como su incorporación a diversos contextos entre los que se encuentra el educativo.

En efecto, los podcast se configuran como una de las herramientas de la Web 2.0 que mejor se han adaptado a entornos educativos. El hecho de que se trate de un instrumento de carácter fundamentalmente sonoro, dada la menor implantación de los podcast de vídeo, ha propiciado su vinculación a aquellas virtualidades de las herramientas pedagógicas basadas en audio como la radio, las grabaciones en casete o CD y, más recientemente, la voz por IP.

Más allá de las potencialidades didácticas de esta herramienta, la creciente utilización de los podcast en el ámbito de la educación y, en particular, de la educación superior se ha visto favorecida fundamentalmente por tres circunstancias: la importante implantación de Internet, la facilidad de acceso a software destinado a la creación y edición digital de archivos de audio y vídeo, y la creciente implantación de dispositivos móviles (iPod, MP3, PDA, etc.) adecuados para el consumo de archivos sonoros (EDUCAUSE, 2006).

Además de dichas circunstancias, cuya motivación es esencialmente tecnológica, resulta destacable otra cuestión de carácter sociológico que ha repercutido positivamente en la expansión de los podcast. la creación y participación de comunidades online. Siguiendo a Edirisngha y Salmon (2007, p.1) "The adoption of Web 2.0 applications - applications, tools and services that enable users to capture, generate and share content and form online communities - has also contributed to the popularity of podcasts". 
Para comprender la relevancia que ha adquirido el podcasting en el ámbito de la educación se ha considerado de interés realizar una aproximación al fenómeno del podcasting educativo y a sus potencialidades, a partir de los trabajos de diversos autores, así como a las primeras iniciativas desarrolladas en este ámbito en Hispanoamérica.

\section{Aproximación al concepto de podcast}

El término podcasting fue acuñado por Ben Hammersley en su artículo "Audible Revolution", publicado en The Guardian; artículo en el que hacía referencia a una nuevo "boom in amateur radio".

"With the Benedit of hindshight, it all seems quite obvious. MP3 players, like Apple's iPod, in many pockets, audio production software cheap or free, and podcast para la docencia en comunicación audiovisual weblogging and established part of the Internet; all the ingredients are there for a new boom in amateur radio. But what to call it? Audioblogging? Podcasting? GuerrillaMedia?" (Hammersley, 2004)

De las denominaciones que Hammersley proponía para dicho fenómeno se terminó adoptando podcasting; término que deriva de la combinación de iPod (nombre comercial del reproductor multimedia de Apple) y Broadcasting (transmitir).

Siguiendo a Tenorio (2008, p. 9), el nacimiento de los podcast es fruto de un proceso que dio comienzo en el año 2000 con el desarrollo, por parte de David Winer, de una nueva etiqueta para la versión 0.92 de RSS (Really Simple Syndication) que permitió la sindicación del primer archivo de audio. En el 
2004 el propio Winer puso fin a este proceso con la creación, con Adam Curry (D) de la cadena MTV, de una aplicación informática para la descarga automática de programas de radio online en el iPod.

De esta manera se puede definir podcasting como un modo de difusión de emisiones radiofónicas que "A través de subscrição de um 'feed RSS', e com a ajuda de um programa específico, pode-se descarregar automaticamente para o computador ou o iPod» (Moura y Carvalho, 2006, p. 88). La existencia de dicho feed permite su diferenciación de cualquier descarga de archivos de sonido colgados en la web.

Esta definición puede completarse, siguiendo a Marcelo y Martín (2007), precisando que los podcast son "programas" que cuentan con diferentes episodios. Un archivo único de audio que permite la descarga desde un sitio web concreto no constituye un podcast en sentido estricto debido a la ausencia de contenido sindicado.

La relevante expansión que han alcanzado los podcast en sus apenas cinco años de existencia ha precisado de una reconceptualización del término que, una vez desligado de la radio online, ha pasado a referirse a una serie de contenidos sin interés comercial que son alojados en Internet, desde donde se pueden consultar y descargar de forma gratuita (Infante, 2006, p. 106).

Entre los diversos ámbitos que se han valido de las potencialidades de los podcast, tanto desde una perspectiva social como tecnológica, resulta destacable el de la educación; ámbito donde se inserta este trabajo.

\section{Posibilidades para la educación}

En el contexto de la educación se ha definido podcasting como "the process of capturing an audioevent, song, speech, or mix of sounds and then posting that 
REVISTA DE LA SEECI.

Piñeiro Otero, Ma Teresa (2009): Podcasting en la educación superior. Una aproximación. No 18. Marzo. Año XIII. Páginas: 129 -147

ISSN: 1576-3420 DOI: http://dx.doi.org/10.15198/seeci.2009.18.129-147

digital sound object to a website or 'blog' in a data structure called an RSS 2.0 envelope (or "feed)" (Meng, 2005, p.1).

Precisamente una de las cuestiones que han favorecido la rápida eclosión del fenómeno podcasting ha sido la posibilidad del oyente de suscribirse a aquellos contenidos que le interesen vía RSS o Atom. Dicha suscripción permite al usuario el acceso automático en su ordenador, o la descarga en su dispositivo portátil, de las actualizaciones de aquellos podcast previamente seleccionados (Kaplan-Leiserson, 2005).

El estudiante que opte por el uso de podcatchers como iTunes o iPodder puede descargar automáticamente dichos contenidos en su ordenador, lo que facilita su acceso, uso y -por tanto- el enriquecimiento del proceso de enseñanzaaprendizaje (Bennington, 2007).

Se puede consumir podcast en cualquier dispositivo digital que permita la reproducción de archivos de audio y multimedia como es el caso de los netbook, MP3, teléfonos móviles de tercera generación (3G), PDA (Personal Digital Assistant), PSP ( Play Station Portable), etc.

"And we watched hundreds of students walking around campus, sitting on the buses, or lying on the grass in the park, with MP3 players 'glued' to their ears. What previously appeared to be a somewhat 'techie' approach to playing music suddenly looked, to us anyway, like something that might have highvalue and low cost for learning" (Salmon y Nie, 2008, p.1).

Pese a constituir un fenómeno muy reciente existe un creciente conjunto de investigaciones en torno al uso de los podcast en el contexto académico Belanger (2005); Campbell (2005); Kaplan-Leiserson (2005); Meng, (2005); 
Cebeci y Tekdal (2006); Hürst y Waizenegger (2006); Chan y Lee (2005); Edirisingha y Salmon (2007); Chan y Lee (2007); Edirisingha y Macharia (2007); Evans (2008); Carvalho, Aguiar, Carvalho y Cabecinhas (2008), entre otros. Trabajos que, no obstante, no cuentan con una base consolidada de investigación (Moss, 2006).

De hecho, atendiendo a Moss (2006), este corpus teórico formado principalmente por artículos en revistas e informes de proyectos acometidos en diferentes instituciones educativas -con especial relevancia de las Universidades- tiende a centrarse en la presencia del fenómeno en la educación. Una focalización que supone una perspectiva más descriptiva, del ejemplo concreto, que analítica.

Dichas investigaciones han destacado las posibilidades de los podcast en el proceso de enseñanza-aprendizaje, especialmente desde la punto de vista de los efectos positivos en los estudiantes, como el enriquecimiento de la experiencia de aprendizaje (Boulos, Maramba y Wheeler, 2006), la potenciación de la atención del estudiante y su reflexión (Baird y Fisher, 2006), el incremento de satisfacción (Miller y Piller, 2005) e, inclusive, la reducción de ansiedad (Chan y Lee, 2005). "Outro aspecto positivo prende-se com o facto de permitir ao professor estar sin-cronizado como os estudantes de hoje, os nado digitais" (Moura y Carvalho, 2006b, p. 2).

Kaplan-Leiserson (2005) diferencia hasta siete utilidades de los podcast en el proceso de enseñanza-aprendizaje:

a. Apoyo sonoro. Los podcast ofrecen un importante apoyo a aquellos estudiantes que prefieren la información oral. La propia grabación de las clases favorece que los estudiantes retengan dicha información. 
b. Otro canal de revisión de material. Los archivos de audio pueden consumirse en momentos de ocio como repaso de los contenidos de la materia, especialmente antes del examen. Para los estudiantes escuchar las clases grabadas supone un refuerzo al curso.

c. Ayuda a los hablantes no-nativos. Les permite revisar las grabaciones tantas veces como sean precisas para entender. El podcasting puede ser de interés para enseñar un lenguaje así como para capturar el discurso y pronunciación de los estudiantes.

d. Un interesante feedback para los estudiantes. Una grabación del profesor que vehicule las correcciones o consideraciones respecto de una actividad desarrollada por el grupo.

e. Cambiar las aulas o sesiones online cuando el contenido requiera de una revisión; los estudiantes pueden acceder siempre y cuando quieran.

f. Dotar de un contenido suplementario, o ser parte de una solución educativa basada en el blended learning. El material puede ser puesto a disposición para el acceso voluntario, o puede ser un componente requerido de una clase o de un curso online.

Estas posibilidades señaladas por Kaplan-Leiserson subrayan las utilidades de los podcast en las diferentes modalidades educativas. Herramienta que va a ser incorporada tanto a una metodología e-learning, a la enseñanza mezclada o semipresencial (b-learning), o como complemento a la enseñanza convencional. En cualquier caso es en el mobile learning ( $m$-learning) donde esta herramienta digital, de esencia sonora, adquiere sus mejores resultados. 


\section{Experiencias pioneras de podcasting en la Educación Superior}

Entre las primeras iniciativas de podcasting en la Educación Superior destaca la llamada Duke Digital Initiative; proyecto que consistió en facilitar a los alumnos del primer año unos iPods para su acceso al material en formato podcast puesto a su disposición por los docentes (Belanguer, 2005). Un material que incluía contenidos de la asignatura concreta, grabaciones de clases (en adelante lecturelearning), grabaciones de campo u otro tipo de elementos complementarios para el estudio. Asimismo, la Universidad de Duke fue la primera institución educativa en alojar un podcast de un congreso universitario, en septiembre de 2005 (Lankshear y Knobel, 2006, p. 176).

Otra de las instituciones pioneras en la integración educativa de los podcast fue la Universidad de Stanford; universidad que puso a disposición de los miembros de su comunidad un canal de iTunes institucional al que poder suscribirse para la descarga de lecturecasting u otro tipo de material complementario para su consumo en el iPod.

En efecto Apple (empresa a la que pertenecen el iPod y el iTunes) ha sido una de las principales impulsoras de este tipo de experiencias educativas especialmente desde la puesta en marcha, junto a diversas universidades de Estados Unidos, de la plataforma "iTunes University". Plataforma desde la que el alumnado puede tener acceso a clases u otro tipo de podcast de carácter docente (Moura y Carvalho, 2006b).

En el ámbito europeo destaca la puesta marcha, en Reino Unido, del Informal Mobile Podcasting and Learning Adaptation (IMPALA); proyecto que comenzó en mayo de 2006 con un programa piloto en la Universidad de Leicester. Este programa permitió subrayar las cualidades del podcasting docente (profcasting) 
para el aprendizaje, especialmente para el aprendizaje autónomo (Salmon y Nie, 2008).

Más allá de estos primeros proyectos desarrollados a partir de la integración didáctica del iPod y del iTunes, la verdadera eclosión de las iniciativas educativas basadas en podcasting en el contexto mundial tuvo lugar a partir del año 2006 (Salmon y Nie, 2008).

\section{Principales iniciativas en el ámbito hispanoamericano}

En el ámbito hispanoamericano los primeros podcast desarrollados en el contexto educativo fueron llevados a cabo en 2005 por Carlos Toledo. Este profesor de Educación General Básica en Chile experimentó con las potencialidades del podcasting educativo, desde la simple grabación y distribución de eventos educativos hasta la puesta en marcha de una verdadera plataforma de contenidos. Una plataforma, denominada Chilepodcast.cl, que funciona como un repositorio de podcast educativos vinculados a los primeros niveles de enseñanza.

Otra de las iniciativas pioneras en el ámbito del podcasting educativo, también en Chile, es el de Educasting. Una iniciativa surgida a partir de un proyecto de tesis realizado por Amir Yazdani-Pedram, Carlos Puccio y Katia Guiloff para la titulación de Comunicación Multimedial, de la Universidad Diego Portales (presentado en septiembre de 2006). Educasting es un proyecto colaborativo enfocado a la correcta creación de los podcast educativos a partir de aquellos parámetros comunicacionales que afectan a la transmisión del conocimiento.

Más concretamente, en el contexto de la Educación Superior, suele considerarse CITRO-UV, desarrollado por el Centro de Investigaciones Tropicales (CITRO) de la Universidad Veracruzana (UV), como el primer podcast académico de divulgación científica en Hispanoamérica. Esta iniciativa, puesta 
en marcha el mes de enero de 2006, consiste en la publicación quincenal de un podcast sobre los avances de la investigación relacionada con el trópico (Escalón, 2006).

En este mismo año, 2006, diversas universidades Hispanoamericanas, conscientes de las potencialidades del podcasting en el contexto educativo tanto desde una perspectiva didáctica como institucional, pusieron en marcha diferentes proyectos de podcast académicos o sobre éstos.

Entre dichas iniciativas destacan aquellas de carácter global, desarrolladas por la propia institución universitaria. Es el caso de la puesta en marcha por la Universidad de San Marcos (Perú) que, en febrero de 2006, publicó su primer Podcast con información institucional.

Otro proyecto en el ámbito de la universidad es el de Podcast UNAM; un servicio de publicación y distribución online de materiales en formato podcast, con fines académicos y científicos.

No obstante también existen iniciativas llevadas a cabo por docentes (de forma individual o en grupo), departamentos o centros concretos. Por ejemplo, la plataforma de contenidos en formato podcast de la Facultad de Comunicaciones de la Universidad Pontificia e Chile. Proyecto liderado por el profesor Francisco Fernández y desarrollado con financiación del Fondo de Desarrollo a la Docencia (FONDEDOC) que surgió con la idea de complementar la web de curso tradicional con una plataforma en la que los estudiantes puedan encontrar podcast con entrevistas a expertos, explicaciones del profesor, sus propias exposiciones, guías de trabajo, etc.

Otros ejemplos de la relevancia que adquirió el fenómeno podcasting en el ámbito de la universidad hispanoamericana durante el año 2006 son las primeras conferencias que incluyen mesas temáticas o workshops vinculados a 
este fenómeno ya desde la perspectiva de la educación, por ejemplo "Technology's Impact on Education" celebrado en la Universidad de Valle de México en septiembre de 2006 (que incluyó entre sus contenidos "Distribution of Educational Content: Podcast in Education"), así como desde una perspectiva más propiamente comunicacional, es el caso de la multiconferencia desarollada en la Universidad de Palermo (Argentina) sobre weblogs, empresas y comunicación ( que contó con una conferencia titulada "Podcasting: una nueva manera de acceder y distribuir contenidos de audio").

A partir de este año, se puede hacer referencia a una importante expansión del fenómeno podcasting en el ámbito de la universidad hispanoamericana. No obstante, siguiendo a Rodríguez Morales (2008), todavía no se puede hablar de una verdadera integración de dichos recursos educativos en la educación universitaria.

\section{Los podcast en la educación. Algunas conclusiones}

La facilidad de acceso y sus posibilidades de uso everywere han favorecido la rápida eclosión del fenómeno podcasting y, más concretamente, su integración en el ámbito de las instituciones educativas.

Esta circunstancia se hace especialmente patente en el ámbito de la Educación Superior donde los podcast se configuran como una herramienta idónea para los diferentes modos de enseñanza-aprendizaje (e-learning, m-learning, $b$ learning), potenciando el aprendizaje autónomo y favoreciendo la flexibilización de las instituciones educativas. 
Tras las primeras iniciativas de podcasting en la Educación Superior, llevadas a cabo en las universidades estadounidenses, la utilización de dicha herramienta se extendió a las instituciones educativas de otros países.

En el contexto hispanoamericano los primeros proyectos de podcast académicos tuvieron lugar apenas un año después de sus primeras manifestaciones en las universidades estadounidenses, aún sin contar con el apoyo de una plataforma como iTunes $U$ (apoyo que tres años después todavía no se ha hecho efectivo). Esta rápida experimentación con el podcasting académico en las instituciones de Educación Superior en Hispanoamérica puede asociarse, de algún modo, a la existencia de una importante tradición de radio universitaria en este contexto.

No obstante, pese a las potencialidades didácticas de los podcast, subrayadas por los resultados de las primeras iniciativas en este ámbito, su implantación en el contexto de las universidades hispanoamericanas se ha desarrollado -con frecuencia- en otras direcciones como la comunicación institucional o la divulgación científica. En este sentido cabe destacar la reciente puesta en marcha de puntoEDU (de UNESCO IESALC), una plataforma de distribución de podcast para la Educación Superior en América Latina y el Caribe que, lejos de constituir una especie de iTunes $U$ latino, tiene como función dotar de contenidos divulgativos a las diferentes radios universitarias de dichos países.

\section{Bibliografía}

BELANGER, Yvonne (2005): "Duke University iPod first year experience final evaluation report". Disponible en: http://cit.duke.edu/pdf/ipod initiative 04 05.pdf (Consultado el 11/10/2008)

BENNINGTON, Adam (2007): "Stick it in your ear: Keeping current with podcasts" enOnline, vol. 31, ㄲo 3. Pág. 30-32. Disponible en: 
REVISTA DE LA SEECI.

Piñeiro Otero, Ma Teresa (2009): Podcasting en la educación superior. Una aproximación. No 18. Año XIII. Páginas: 129 -147

ISSN: 1576-3420 DOI: http://dx.doi.org/10.15198/seeci.2009.18.129-147

http://www.allbusiness.com/technology/softwareservices-

applications/10590301-1.html Consultado el 11/10/2008)

BOULOS, Maged, MARAMBA, Inocencio y WHEELER, Steve (2006): "Wikis, blogs and podcasts: a new generation of Web-based tools for virtual collaborative clinical practice and education", en BMC Medical Education, vol. 41, ㄲo 6. Disponible en: http://www.biomedcentral.com/content/pdf/1472-6920-6-41.pdf (Consultado el 23/06/2008)

CAMPBELL, Gardner (2005): "There's something in the air: Podcasting in education" en EDUCAUSE Review, vol. 40, № 6. Pág. 32-47. Disponible en: http://www.educause.edu/apps/er/erm05/erm0561.asp (Consultado 07/10/2008)

CARVAlHo, Ana A., AGUIAR, Cristina, CARVALHO, Carla J. y CABECINHAS, Rosa (2008): "Influence of podcasts characteristics on higher students" acceptance" en BONK, Curtis y otros: Proceedings of E-Learn. Chesapeake, AACE. Pág. 3625-3633. Disponible en: http://www.editlib.org/noaccess/30190 (Consultado el 21/12/2008)

CARVALHO, Ana A.; CRUZ, Sonia y MOURA, Adelina (2008b): "Pedagogical potentialities of podcasts in learning-reactions from $k-12$ to university students in Portugal" en WHEELER, Steve y otros: Conference Proceedings of LYICT 2008. Kuala Lumpur, IFIP and Open University of Malaysia. Pág. 23-32. Disponible en: http://dl.ifip.org/iojs/index.php/ifip/article/view/13557 (Consultado el 21/12/2008)

CEBECI, Zeynel y TEKDAL, Mehmet (2006): "Using podcasts as audio learning objects" en Interdisciplinary Journal of Knowledge and Learning Objects, ํo 2. 
Pág. 7-57. Disponible en: http://ijklo.org/Volume2/v2p047-057Cebeci.pdf (Consultado el 11/06/2008).

CEBECl, Zeynel y TEKDAL, Mehmet (2006): "Using podcasts as audio learning objects" en Interdisciplinary Journal of Knowledge and Learning Objects, vol. 2. Pág. 7-57. Disponible en: http://ijklo.org/Volume2/v2p047-057Cebeci.pdf (Consultado el 17/06/2008)

CHAN, Anthony y LEE, Mark (2007): "Reducing the effects of isolation and promotion inclusivity for distance learners throught podcasting" en Turkish Online Journal of Distance Education-TOJDE, vol. 8, no1. Pág. 85-104. Disponible en: http://tojde.anadolu.edu.tr/tojde25/pdf/article_7.pdf (Consultado el $17 / 09 / 2008)$

CHAN, Anthony y LEE, Mark (2005): "An MP3 a day keeps the worries away. Exploring the use of podcasting to address preconceptions and alleviate preclass anxiety amongst undergraduate information technology students" en Student Experience Conference, Wagga Wagga, New South Wales, 5-7 de septiembre Disponible en: http://www.csu.edu.au/division/studserv/sec/papers/chan.pdf> (Consultado el $11 / 10 / 2008)$

EDIRISINGHA, Palitha y MACHARIA, Raymonf (2007): "Podcasting to support learning in Higher Education adopting IMPALA models" en Podcasting workshop, Nairobi, 31 mayo de 2007. Disponible en:

http://www2.le.ac.uk/projects/impala/presentations/impala-workshop-at-theuniversity-of-nairobi-

kenya/Impala University\%20of\%20Nairobi 31May2007.pdf (Consultado el 10/09/2008) 
REVISTA DE LA SEECI.

Piñeiro Otero, Ma Teresa (2009): Podcasting en la educación superior. Una aproximación. No 18. Marzo. Año XIII. Páginas: 129 -147

ISSN: 1576-3420 DOI: http://dx.doi.org/10.15198/seeci.2009.18.129-147

EDIRISINGHA, Palitha y SALMON, Gilly (2007): "Pedagogical modeld for podcast in higher education" en Beyond Distance Research Alliance Conference pre-print copy, Mayo 2007. Disponible en: https://lra.le.ac.uk/handle/2381/405 (Consultado el 24/08/2010).

EDUCAUSE (2006): The Horizon Report, 2006. The New Media. Austin.

ESCALÓN, Edith (2006): "Lanza CITRO-UV el primer Podcast de divulgación científica en $A L^{\prime \prime}$ en Gaceta Universidad Veracruzana, Enero-Marzo, № 97. Disponible en: http://www.uv.mx/gaceta/Gaceta\% 20virtual\% 2097/Gaceta97/97/ABCiencia/AB Ciencia06.htm (Consultado el 01/07/2008)

EVANS, Chris (2008): "The effectiveness of m-learning in the form of podcast revision lectures in higher education" en Computers \& Education, vol. 50. Pág. 491-498.

GEOGHEAN, Michael y KLASS, Dan (2005): Podcast solutions: the complete guide to podcasting. Friendsoft. Nueva York.

HAMMERSLEY, Brian (2004): "Audible revolution" en The Guardian, 12 de febrero de 2004, Londres. Disponible en: http://www.guardian.co.uk/media/2004/feb/12/broadcasting. digitalmedia (Consultado el 22/02/2008)

HÜRST, Wolfgang y WAIZENEGGER, Wolfgang (2006): "An overview of different approaches for lecture casting" en ISAÍAS, Pedro y otros: Proceedings of IADIS International Conference on Mobile Learning 2006, Dublin, International Association for Development of the Information Society. Disponible en: http://www.iadis.net/dl/final_uploads/200605F008.pdf (Consultado el 22/02/2008) 
INFANTE, I sabel (2006): "Emissões Livres" en Revista Exame Informática, vol. 130, Oporto, Medipress. Pág. 106-109.

KAPLAN-LEISERSON, Eva (2005): "Podcasting in academic and corporate learning" en Learning circuits. American Society for Training \& Development. Disponible en: http://www.astd.org/LC/2005/0605 kaplan.htm (Consultado el 22/08/2008)

Lankshear, Colin y Knobel, Michele (2006): New literacies>: Everyday Practices \& Classroom learning. McGraw-Hill Education. Nueva York.

MARCELO, Juan Francisco y MARTín, Eva Rosario (2007): Podcastig. Anaya Multimedia. Madrid.

MENG, Peter (2005): "Podcasting and vodcasting: A white paper. Definitions, discussions, andimplications" Disponible en: http://edmarketing.apple.com/adcinstitute/wpcontent/Missouri Podcasting Whi te Paper.pdf (Consultado el 12/03/2008)

MILLER, Michael y PILLER, Michael (2005): "Principal factors of an audio reading delivery mechanism evaluating educational use of the iPod" en KOMMERS, Piet y RICHARDS, Griff : Proceedings of world conference on educational multimedia, hypermedia and telecommunications. Chesapeke, AACE. Pág. 260-267. Disponible en: http://www.editlib.org/d/20091/proceeding 20091.pdf (Consultado el 11/07/2008)

MOSS, Nathan (2006): "Open the Pod Bay Doors" en Directions in education: an independent policy advisory service for leaders in education, vol. 14, № 20. 
REVISTA DE LA SEECI.

Piñeiro Otero, Ma Teresa (2009): Podcasting en la educación superior. Una aproximación. No 18. Marzo. Año XIII. Páginas: 129 -147

ISSN: 1576-3420 DOI: http://dx.doi.org/10.15198/seeci.2009.18.129-147

Disponible en: http://eprints.qut.edu.au/7759/1/7759.pdf (Consultado el $12 / 10 / 2008)$.

MOURA, Adelina y CARVALHO, Ana Amélia (2006): "Podcast: Potencialidades na Educação" en Prisma.com, vol. 3. Pág. 88-120. Disponible en: http://portal.doc.ua.pt/journals/index.php/prismacom/article/viewFile/623/pdf (Consultado el 11/07/2008)

MOURA, Adelina y CARVALHO, Ana Amélia (2006b): "Podcast: Uma ferramenta para usar dentro e fora da sala de aula" en JOSÉ, Rui y BAQUERO, Carlos: Proceedings of the Conference on Mobile and Ubiquitous Systems. Guimarães, Universidade do Minho. Pág. 155-158. Disponible en: http://adelinamouravitae.com.sapo.pt/ubiquitouspodcast.pdf (Consultado el $11 / 07 / 2008)$

PENNINGTON, Adam (2007): "Stick it in your ear: Keeping current with podcasts" en Online, vol. 31, № 3. Pág. 30-32. Disponible en: http://www. allbusiness.com/technology/software-services-

applications/10590301-1.html (Consultado el 10/09/2008)

RODRÍGUEZ MORALES, Germania (2008): "Educación Superior en Latinoamérica y la Web 2.0" Disponible en: http://www.virtualeduca.info/ponencias2009/172/webyeducacion.pdf (Consultado el 10/12/2008)

SALMON, Gilly y NIE, Ming. (2008): "Doubling the life of iPods" en SALMON, Gilly y EDIRISINGHA, Palitha: Podcasting for learning in universities. McGrawhill. Reino Unido.

SCANLON, Eileen, JONES, Ann y WAYCOTT, Jenny (2005): "Mobile technologies: prospects for their use in learning in informal science settings" en 
REVISTA DE LA SEECI.

Piñeiro Otero, Ma Teresa (2009): Podcasting en la educación superior. Una aproximación. No 18. Marzo. Año XIII. Páginas: 129 -147

ISSN: 1576-3420 DOI: http://dx.doi.org/10.15198/seeci.2009.18.129-147

Journal of Interactive Media in Education, vol. 25. Pág. 1-17. Disponible en: http: //jime.open.ac.uk> (Consultado el 15/09/2008)

TENORIO, I ván (2008): Podcast. Manual del podcaster. Marcombo. Barcelona.

\section{Webgrafía}

http://podcastellano.es

http://www. educasting.info

http://chilepodcast.cl/ 